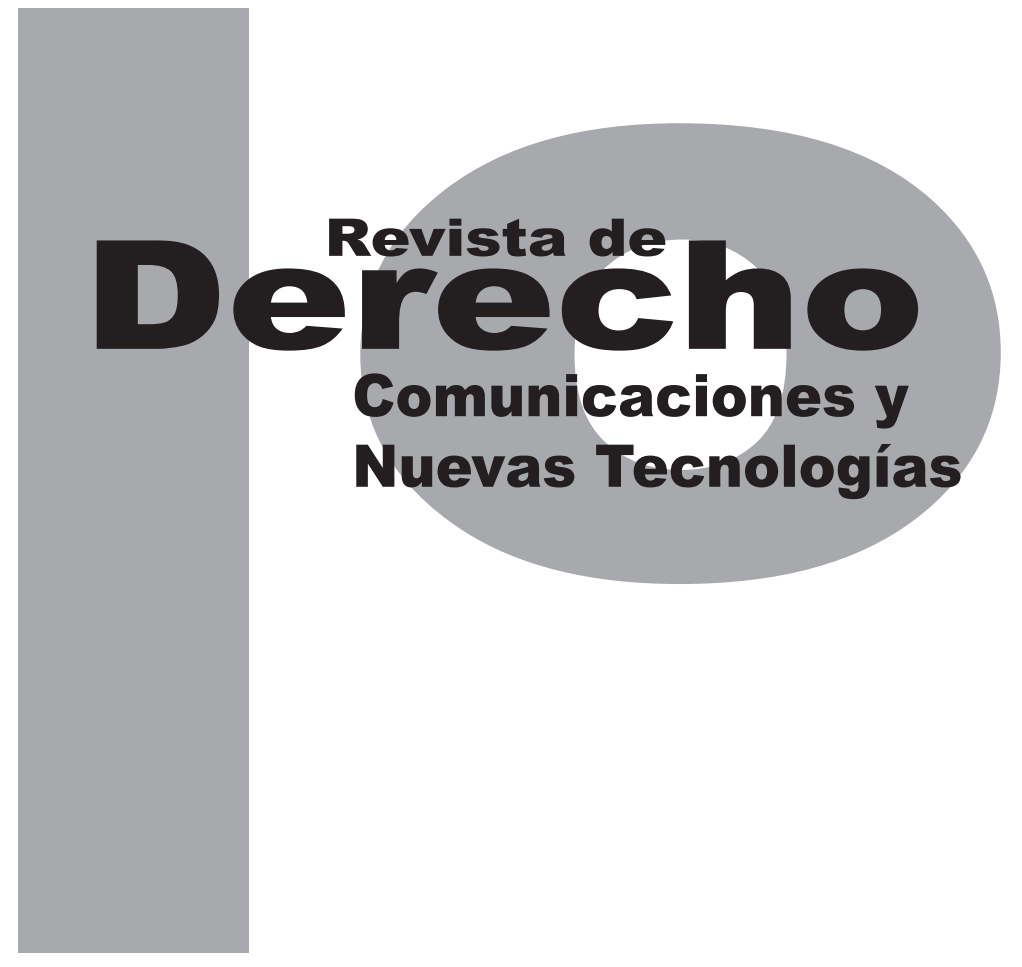

\title{
REGLAMENTACIÓN DEL USO DEL ESPECTRO RADIOELÉCTRICO EN LAS ACTIVIDADES ESPACIALES
}

\author{
ana María Palacio Jaramillo
}

Artículo de reflexión

DOI: http://dx.doi.org/10.15425/redecom.14.2015.10

Universidad de los Andes

Facultad de Derecho

Rev. derecho comun. nuevas tecnol.

No. 14, julio - diciembre de 2015. ISSN 1909-7786 


\section{Reglamentación del uso del espectro radioeléctrico en las actividades espaciales}

\section{Resumen}

Desde la prehistoria, la comunicación ha tenido una enorme importancia en el desarrollo social del ser humano. No solo constituye la base de la sociedad y de las relaciones sociales entre los individuos y su entorno, sino que representa una importante influencia para el progreso de las comunidades. Los métodos y canales de comunicación a lo largo de la historia han venido evolucionando y aumentando cada vez con mayor velocidad hasta llegar al estudio y desarrollo del espacio ultraterrestre. Es por esto que el presente artículo pretende explicar cómo ha sido el desarrollo de las telecomunicaciones en la comunidad internacional, y específicamente lo relevante respecto a la distribución del espectro electromagnético y las frecuencias radioeléctricas.

Palabras clave: comunicación, telecomunicaciones, espectro radioeléctrico, frecuencias radioeléctricas, espacio ultraterrestre, segunda guerra mundial, guerra fría, órbita geoestacionaria, Unión Internacional de Telecomunicaciones.

\section{Regulation of the use of radio electric spectrum in space activities}

\section{Abstract}

Since prehistoric times communication has had an enormous importance on the social development of human beings. Not only is it the basis of society and social relationships between individuals and their environment, it represents an important influence on community progress. The methods and channels of communication throughout history have been evolving and growing with increasing speed up until the study and development of outer space. As such this article aims to explain the development of telecommunications in the international community, specifically with regard to relevant aspects of the distribution of the electromagnetic spectrum and radio frequencies.

Keywords: communication, telecommunications, radio electric spectrum, radio electric frequencies, outer space, World War II, Cold War, geostationary orbit, International Telecommunication Union.

\section{Regulamentação do uso do espectro radioelétrico nas atividades espaciais}

\section{Resumo}

Desde a pré-história, a comunicação tem tido uma enorme importância no desenvolvimento social do ser humano. Não só constitui a base da sociedade e das relações sociais entre os indivíduos e seu entorno, senão que representa uma importante influência para o progresso das comunidades. Os métodos e canais de comunicação ao longo da história têm vindo evoluindo e aumentando cada vez com maior velocidade até chegar ao estudo e desenvolvimento do espaço ultraterrestre. É por isto que o presente artigo pretende explicar como foi o desenvolvimento das telecomunicações na comunidade internacional, e especificamente o que é relevante com respeito à distribuição do espectro eletromagnético e as frequências radioelétricas.

Palavras-chave: comunicação, telecomunicações, espectro radioelétrico, frequências radioelétricas, espaço ultraterrestre, segunda guerra mundial, guerra fria, órbita geoestacionária, União Internacional de Telecomunicações. 


\title{
Reglamentación del uso del espectro radioeléctrico en las actividades espaciales*
}

\author{
Ana María Palacio Jaramillo**
}

\section{SUMARIO}

Introducción - I. DEFINICIONES GENERALES DE ESPECTROS, BANDAS Y ONDAS - A. Espectro electromagnético - $B$. Espectro radioeléctrico - II. NORMAS INTERNACIONALES APLICABLES AL MANEJO DEL ESPECTRO ELECTROMAGNÉTICO Y DEL ESPECTRO RADIOELÉCTRICO - A. Constitución y Convenio Internacional de Telecomunicaciones y creación de la Unión Internacional de Telecomunicaciones - B. Tratado de Montreux de 1965 y Conferencia de Plenipotenciarios de la Unión Internacional de Telecomunicaciones (Málaga-Torremolinos 1973) - C. Reglamento de las Radiocomunicaciones de la UIT de 1992 - D. Tratado de Nairobi de 1982 - E. Enmiendas a la Constitución de la UIT - III. NORMAS NACIONALES APLICABLES AL MANEJO DEL ESPECTRO ELECTROMAGNÉTICO Y DEL ESPECTRO RADIOELÉCTRICO - A. Agencia Nacional de Espectros - IV. CONCLUSIONES - Referencias.

* Cómo citar este artículo: Palacio Jaramillo, A. M. (Diciembre, 2015). Reglamentación del uso del espectro radioeléctrico en las actividades espaciales. Revista de Derecho, Comunicaciones y Nuevas Tecnologías, 14. Universidad de los Andes (Colombia).

** Estudiante de noveno semestre de Derecho de la Universidad de los Andes. Monitora de la cátedra de Derecho del Espacio Ultraterrestre. Correo: am.palacio1233@uniandes.edu.co 
Introducción

Desde la prehistoria, la comunicación ha tenido una enorme importancia en el desarrollo social del ser humano. No solo constituye la base de la sociedad y de las relaciones sociales entre los individuos y su entorno, sino que representa una importante influencia para el progreso de las comunidades. Los métodos y canales de comunicación a lo largo de la historia han venido evolucionando y aumentando cada vez con mayor velocidad, pues la necesidad del ser humano de comunicarse ha estado presente desde el origen de la especie, como lo muestran las pinturas y símbolos utilizados desde épocas prehistóricas. Esta urgencia del hombre por descubrir nuevos métodos que permitieran una comunicación a distancia y en tiempo real, ocasionó un importante avance en materia de comunicaciones durante el siglo xix. Teniendo ya el alfabeto, la imprenta y las cartas, fue en 1837 con el descubrimiento del telégrafo cuando se obtuvo por primera vez alguna forma de comunicación eléctrica. Este importante descubrimiento sentó las bases para posteriores avances como el teléfono, el televisor e incluso el celular. Gracias a la rápida adopción del telégrafo por parte de la comunidad europea e internacional, surgió la necesidad de crear acuerdos de interconexión de las redes telegráficas nacionales e internacionales. Estos acuerdos fueron consagrados en el Convenio Telegráfico Internacional de 1865, que en el año de 1932 se fusionó con el Convenio Internacional de Radiotelegrafía de 1906 para crear lo que se conoce actualmente como el Convenio Internacional de las Telecomuni- caciones. Este último constituye el documento fundador de la Unión Internacional de Telecomunicaciones (UIT), entidad que actualmente es responsable de la atribución del espectro de frecuencias radioeléctricas y las órbitas de satélite, y de la normalización y el desarrollo de las tecnologías de la información y la comunicación (TIC) en la comunidad internacional.

De igual forma, es claro que el avance de las comunicaciones se encuentra fuertemente ligado al estudio y desarrollo del espacio ultraterrestre. Al respecto es importante hacer referencia a la teoría planteada por el científico británico Arthur Clarke sobre de la existencia de la órbita de los satélites geoestacionarios y sus características. Esta teoría plantea que la órbita geoestacionaria se encuentra a una distancia de $35.875 \mathrm{ki}-$ lómetros sobre la línea ecuatorial de la Tierra, en donde hay una unión de la fuerza centrífuga producida por nuestro planeta y la fuerza propagada en el vacío, que causa que cualquier objeto ubicado en ella gire a la misma velocidad de la Tierra. Por consiguiente, si se ubica un satélite en esta órbita, un observador desde la Tierra podría pensar que este está inmóvil. Como consecuencia, en octubre de 1945 Clarke propuso la posibilidad de establecer la comunicación satelital a través de canales de teléfono y de radiodifusión dependientes únicamente de los satélites, y en particular de aquellos ubicados en la órbita geoestacionaria. Esto representó un descubrimiento indispensable para el desarrollo de las telecomunicaciones como las conocemos hoy en día. 
En las últimas décadas del siglo xx hemos sido testigos de la expansión de los sistemas de comunicaciones inalámbricos, desde los teléfonos celulares hasta la televisión, los sistemas de radiocomunicaciones inteligentes y la comprobación técnica del espectro, entre otros. La tecnología de las radiocomunicaciones se ha convertido en un elemento esencial en materia de servicios públicos fundamentales, tales como los sistemas de navegación por satélite, el control medioambiental e incluso la investigación en el espacio ultraterrestre.

Lo que ha hecho posible este rápido avance en materia de telecomunicaciones ha sido el desarrollo y distribución del espectro electromagnético y las frecuencias radioeléctricas en la comunidad internacional. Por esta razón, en el presente trabajo se explicará el desarrollo que ha tenido la reglamentación del espectro radioeléctrico y la importancia de darle una utilización racional, equitativa, eficaz y económica, para lo cual se tendrá en cuenta la siguiente estructura: (i) definiciones generales de espectros, bandas y ondas radioeléctricas, (ii) normas internacionales aplicables al manejo del espectro electromagnético y del espectro radioeléctrico y (iii) normas nacionales aplicables al manejo del espectro electromagnético y del espectro radioeléctrico.

\section{DEFINICIONES GENERALES DE ESPECTROS, BANDAS Y ONDAS}

\section{A. Espectro electromagnético}

El espectro electromagnético es un campo energético compuesto de ondas electromagnéticas ${ }^{1}$ con diferentes frecuencias o longitudes. Las ondas electromagnéticas se propagan mediante una oscilación de campos eléctricos y magnéticos, produciendo así una radiación electromagnética que se extiende a través del espacio transportando energía de un lugar a otro. Esta radiación puede manifestarse por medio de calor, luz visible, ondas de radio, televisión, telefonía, etc., y es medida en hercios $(\mathrm{Hz})$, la unidad internacional de medida del espectro electromagnético.

La energía proveniente de la radiación se manifiesta en ondas que se pueden difundir a través de distintos medios, es decir, no necesitan de ningún medio especial (aire, agua) para su propagación, lo que significa que pueden hacerlo en el vacío y a la velocidad de la luz. Además, las ondas pueden tener diferentes frecuencias; esto es, no todas cuentan con los mismos intervalos de repetición. Entonces, entre más alta sea una frecuencia mayores repeticiones tendrá una onda electromagnética determinada.

El hecho de que estas ondas puedan propagarse en el vacío ha permitido su utilización

Estas ondas electromagnéticas fueron descubiertas por el matemático y físico James Clerk Maxwell a partir de un análisis cuidadoso de las ecuaciones del campo electromagnético. Posteriormente, Heinrich Hertz calculó la forma de las ondas a partir de las ecuaciones de Maxwell, para un espacio vacío en el que no intervienen cargas ni corrientes. 
en el espacio ultraterrestre, especialmente en lo relacionado con los sistemas satelitales de telecomunicación. Sin embargo, cuando estas se propagan en el espacio pueden surgir varias complicaciones, al ser afectadas por algunos fenómenos físicos dentro de los que se encuentran: (i) la reflexión, (ii) la refracción, (iii) la difracción, (iv) la interferencia y (v) el efecto doppler.

Por un lado, la reflexión ocurre cuando una onda cambia su dirección al encontrarse con un nuevo medio que no puede atravesar. Por otro lado, la refracción se presenta cuando una onda cambia de dirección al entrar en un nuevo medio en el que viaja a una velocidad distinta; y la difracción se da cuando una onda, al topar con el borde de un obstáculo, lo rodea, y por lo tanto ya no se mueve en línea recta.

La interferencia tiene lugar cuando dos ondas se combinan al encontrase en el mismo punto del espacio. Esta puede ser entendida como:

El efecto de una energía no deseada debida a una o varias emisiones, radiaciones, inducciones o sus combinaciones sobre la recepción en un sistema de radiocomunicación, que se manifiesta como degradación de la calidad, falseamiento o pérdida de la información que se podría obtener en ausencia de esta energía no deseada. (UIT, 1985).

Finalmente, el efecto doppler es el resultado del movimiento relativo entre la fuente emisora de las ondas y el receptor de estas (Rincón, 2013, pág. 18). Es claro entonces que el espectro radioeléctrico está dado para medir frecuencias, en la medida en que está compuesto por luz y radiación electromagnética.

\section{B. Espectro radioeléctrico}

Ahora bien, el espectro radioeléctrico es el medio por el cual se transmiten las frecuencias de ondas de radio electromagnéticas que permiten las telecomunicaciones (radio, televisión, Internet, telefonía móvil, televisión digital terrestre, etc.).

Ha sido definido por la UIT, organismo especializado de las Naciones Unidas para las TIC, como:

Las frecuencias del espectro electromagnético usadas para los servicios de difusión y servicios móviles, de policía, bomberos, radioastronomía, meteorología y fijos. Este '(...) no es un concepto estático, pues a medida que avanza la tecnología se aumentan (o disminuyen) rangos de frecuencia utilizados en comunicaciones, y corresponde al estado de avance tecnológico'. (Uit, 1985).

En el mismo sentido, la Agencia Nacional del Espectro (ANE) afirma que el espectro radioeléctrico es el "medio por el cual se transmiten las frecuencias de ondas de radio electromagnéticas que permiten las telecomunicaciones y son administradas y reguladas por los gobiernos de cada país." (2011, párr. 1).

El espectro radioeléctrico, al igual que el espectro electromagnético, está compuesto por ondas, cada una de ellas con diferentes frecuencias. Por esto, el espectro radioeléctrico cuenta con una variedad de bandas de frecuencias, di- 
vididas a su vez en frecuencias terrestres y en las frecuencias satelitales. ${ }^{2}$

El espectro radioeléctrico se encuentra dividido en diferentes bandas de frecuencia que permiten la distribución de las distintas frecuencias electromagnéticas en los diversos servicios de telecomunicaciones que se deben prestar. En otras palabras, las bandas de frecuencia del espectro radioeléctrico que se trasladan de un lugar a otro sirven para el desarrollo de distintos tipos de telecomunicaciones, como la transmisión de radio, la televisión por cable, entre otros. Es decir, la longitud de la onda electromagnética es determinante para establecer el uso específico que se le dará, pues este depende de la frecuencia determinada en la que sea utilizado.

La ANE ha definido las diferentes bandas de la siguiente manera:

1. Banda UHF: se trata de un rango de frecuencia en el cual se ubican las ondas electromagnéticas que son utilizadas por las compañías de telefonía fija y telefonía móvil, distintas compañías encargadas del rastreo satelital de automóviles y establecimientos, y las emisoras radiales como tal.

2. Banda vHF: es una banda más potente que puede llegar a tener un alcance mayor, incluso a nivel internacional. También es utilizada por las compañías de telefonía móvil y terrestre, las emisoras radiales y los sistemas de telefonía móvil en aparatos voladores.

2 Acorde con la ubicación de las bandas dentro del espectro radioeléctrico, las características naturales de esa banda definen las aplicaciones que en ellas pueden explotarse.
3. Banda HF: banda de alta frecuencia que produce una mayor cobertura de transmisión. Es utilizada para comunicaciones de aviación, radares y algunos casos de telefonía móvil y marina. (ANE, 2011).

De acuerdo con lo anterior, es claro que cada banda o región presenta características especiales que determinan el tipo de servicio específico que se puede prestar en el mercado. Al poseer diferentes propiedades, las longitudes de onda van a permitir la ejecución de distintas actividades. Por lo tanto, resulta necesario regular la materia y crear un sistema de coordinación internacional por medio de protocolos y normas internacionales, que permitan a su vez reglamentar la materia en el plano nacional.

\section{NORMAS INTERNACIONALES APLICABLES AL MANEJO DEL ESPECTRO ELECTROMAGNÉTICO Y DEL ESPECTRO RADIOELÉCTRICO}

Teniendo en cuenta lo anterior, es claro que cada Estado debe implementar procedimientos para garantizar la correcta gestión, vigilancia y control del espectro radioeléctrico. La gestión del espectro radioeléctrico (o de frecuencias) se puede entender como la combinación de los procedimientos jurídicos, económicos, científicos, administrativos y técnicos necesarios para garantizar el correcto funcionamiento de los canales radioeléctricos. Se debe garantizar que estos canales puedan ser utilizados por las estaciones de los distintos servicios de radiocomunicaciones, en cualquier banda del espectro 
y en cualquier momento dado, sin producir ni recibir interferencias perjudiciales.

Dentro de las facultades de gestión, administración y control del espectro electromagnético se encuentran las actividades de planeación y coordinación, así como la fijación del cuadro nacional de frecuencias para garantizar una efectiva asignación y otorgamiento de permisos para su utilización. Además, se debe garantizar la protección y defensa del espectro radioeléctrico por medio de la comprobación técnica de emisiones radioeléctricas, el establecimiento de condiciones técnicas para su utilización y la adopción de medidas tendientes a establecer el correcto y racional uso del espectro radioeléctrico, y a restablecerlo en caso de perturbación o irregularidades (ANE, 2011).

La gestión, vigilancia y control del espectro electromagnético ha tenido un desarrollo progresivo en el derecho internacional del derecho ultraterrestre, pues ha sido un tema importante en diferentes tratados, convenios y resoluciones internacionales. Por esta razón se hará una explicación de cada uno para analizar su influencia en el tema del espectro radioeléctrico y su utilización por parte de los diferentes Estados. En primer lugar, se hará referencia a la Constitución y el Convenio Internacional de Telecomunicaciones por medio de la cual se creó la uit. Luego se analizarán los diferentes tratados y convenios relevantes, como son: (i) el Tratado de Montreux de 1965, (ii) el Tratado de Nairobi de 1982, (iii) el Reglamento de las Radiocomunicaciones de la UIT de 1992 y (iv) finalmente las enmiendas que se han realizado a la Constitución de la uाT en la Conferencia de Plenipotenciarios de Kyoto de 1994, la Conferencia de Minneapolis de 1998 y la Conferencia de Marrakech en 1998.

\section{A. Constitución y Convenio Internacional de Telecomunicaciones, y creación de la Unión Internacional de las Telecomunicaciones}

\section{Historia y creación de la UIT}

Antes de explicar el desarrollo de la reglamentación sobre el uso del espectro radioeléctrico en la comunidad internacional, es importante conocer acerca de la historia, la creación y el desarrollo de la UIT, el organismo especializado de las Naciones Unidas para las tecnologías de la información y la comunicación.

Como tal, es responsable de la atribución del espectro de frecuencias radioeléctricas y las órbitas de satélite, y de la normalización y el desarrollo de las tic en la comunidad internacional. La UIT se ha encargado de elaborar normas técnicas que garantizan la interconexión continua de las redes y las tecnologías, con el objetivo de garantizarles a los diferentes Estados, sin importar su nivel de desarrollo, el derecho fundamental a la comunicación.

La uIT se creó en un momento en el cual el desarrollo tecnológico estaba apenas comenzando y avanzaba cada vez con mayor velocidad. Con el invento del telégrafo y su rápida acogida por la comunidad internacional surgió la necesidad de crear acuerdos bilaterales y multilaterales entre los diferentes Estados para regular los 
canales y métodos de comunicación. Así las cosas, gracias a la iniciativa de veinte Estados europeos se organizó una reunión en la cual se tenía como principal propósito desarrollar un acuerdo marco para la interconexión internacional. Por esto, se estableció el Convenio Telegráfico Internacional de 1865, en el cual se acordó una normatividad uniforme para facilitar la interconexión internacional, se establecieron normas universales para la explotación y se fijaron tarifas aplicables a todos los países.

Sin embargo, en 1932 la comunidad internacional decidió fusionar el Convenio Telegráfico Internacional de 1865 y el Convenio Internacional de Radiotelegrafía de 1906 en el Convenio Internacional de las Telecomunicaciones, que constituye el origen de la Unión Internacional de las Telecomunicaciones. En el año de 1947, esta institución se hizo parte de la Organización de las Naciones Unidas, mediante un acuerdo aprobado por la Asamblea General de la onu el día 15 de noviembre de 1947.

\subsection{La UIT en la actualidad}

La Constitución de la uit establece en su artículo $1^{\circ}$ las principales funciones que debe desempeñar, a saber: la "atribución de las bandas de frecuencias del espectro radioeléctrico y la adjudicación de las frecuencias radioeléctricas." De igual forma, debe llevar el registro de las asignaciones de frecuencias y las posiciones orbitales asociadas en la órbita de los satélites geoestacionarios, a fin de evitar toda interferencia perjudicial entre las estaciones de radiocomunicación de los distintos países. Así mismo, le corresponde prevenir las interferencias perjudiciales entre las estaciones de radiocomunicación de los diferentes países y mejorar la utilización del espectro de frecuencias radioeléctricas y de la órbita de los satélites geoestacionarios. De acuerdo con lo anterior, puede afirmarse que una de las principales funciones de la uit corresponde a la regulación del espectro radioeléctrico y de las posiciones orbitales para garantizar su uso eficaz y equitativo en materia de servicios de telecomunicaciones. Para esto, creó un Reglamento de las Radiocomunicaciones que se analizará más adelante.

Ahora bien, en cuanto a su conformación, la Uiт está integrada por miembros tanto del sector público como del sector privado. Cuenta con 193 Estados Miembros, como también con más de 560 Miembros de Sector ${ }^{3}$ y organismos reguladores de las Tic, instituciones académicas y de investigación, y unas 700 empresas privadas. Lo anterior evidencia que la uाt se fundamenta en el principio de cooperación internacional entre los gobiernos de los Estados Miembros y el sector privado.

La Uit cumple su objetivo fundamental por medio de tres sectores principales: (i) el Sector de Radiocomunicaciones (UIT-R), (ii) el Sector de Normalización de las Telecomunicaciones (UIT-T) y (iii) el Sector de Desarrollo de las Telecomunicaciones (UIT-D).

3 Estos participan en los trabajos de un sector determinado ( $R$, $T$ o D). Entre ellos cabe citar a las empresas de explotación, las organizaciones científicas o industriales, las instituciones financieras o de desarrollo, otras entidades que se ocupan de asuntos relativos a las telecomunicaciones y otros organismos internacionales de telecomunicaciones, de normalización, financieros o de desarrollo. 
El Sector de Radiocomunicaciones (UIT-R) se encarga de la atribución de frecuencias radioeléctricas, la gestión de las órbitas de los satélites y la regulación de las tecnologías de acceso. De igual forma, desempeña un papel vital en la elaboración de normas presentadas en forma de Recomendaciones UIT-R, destinadas a la gestión del espectro radioeléctrico; adicionalmente tiene la labor de coordinar y asegurar que los satélites de comunicaciones, radiodifusión y meteorología, que giran alrededor de la Tierra, puedan coexistir sin que ninguno de ellos cause interferencia perjudicial a los servicios de otro.

La especialidad del Sector de Radiocomunicaciones de la uाt consiste en favorecer la colaboración internacional, a fin de asegurar la utilización racional, equitativa, eficaz y económica del espectro de frecuencias radioeléctricas y de las órbitas de los satélites, mediante: (i) la celebración de conferencias mundiales y regionales de radiocomunicaciones, (ii) la formulación de Recomendaciones UIT-R, (iii) la coordinación de la labor encaminada a suprimir las interferencias perjudiciales entre estaciones radioeléctricas de diferentes países, (iv) la actualización del Registro Internacional de Frecuencias y (v) la organización de seminarios para contribuir a la gestión del espectro de frecuencias radioeléctricas a escala nacional e internacional.

Al Sector de Normalización de las Telecomunicaciones (UIT-T) le corresponde la parte normativa, es decir, las Recomendaciones, las cuales son fundamentales para el funcionamiento de las redes TIC actuales en el plano internacional.
El Sector de Desarrollo de las Telecomunicaciones (UIT-D) cuenta con diferentes programas que permiten a los Estados entrar o aumentar su presencia en nuevos mercados, aprender a aplicar prácticas idóneas en materia de tıc, entre otros desarrollos importantes para facilitar el acceso y progreso en el sector.

En este sentido, es claro que la uाt lleva a cabo una importante labor de gestión del espectro de frecuencias radioeléctricas, al garantizar que los servicios definidos en el Reglamento de Radiocomunicaciones se utilicen de manera eficaz y que su explotación no cause interferencias perjudiciales a los demás servicios radioeléctricos. Además, se encarga de regular los principios y técnicas de la gestión del espectro al plantear estrategias a largo plazo respecto a su utilización, al definir los planteamientos económicos de la gestión nacional y al llevar a cabo los planes de asistencia a los países en desarrollo, en cooperación con el Sector de Desarrollo de las Telecomunicaciones.

\section{B. Tratado de Montreux de 1965 y Conferencia de Plenipotenciarios de la Unión Internacional (Málaga-Torremolinos 1973)}

El Tratado de Montreux de 1965 es el primer desarrollo normativo en lo referente al uso del espectro radioeléctrico. En su artículo 46 establece:

Los Miembros y Miembros asociados reconocen la conveniencia de limitar el número de las frecuencias y el espacio del espectro utilizados al mínimo indispensable para asegurar de manera satisfactoria el funcionamiento de los servi- 
cios necesarios. A tales fines, será conveniente que se apliquen, a la mayor brevedad, los adelantos técnicos más recientes.

Es claro, entonces, que el uso y desarrollo de este espectro debe ser concebido y analizado desde el punto de vista de las telecomunicaciones y el derecho internacional del espacio ultraterrestre.

Ahora bien, en la Conferencia de Plenipotenciarios de la Unión Internacional de Telecomunicaciones (UIT) de Málaga-Torremolinos, celebrada en España en 1973, se le dio al espectro radioeléctrico el tratamiento de recurso natural limitado pero no agotable; en cuanto al uso, gestión y acceso a la órbita y las frecuencias radioeléctricas, se estableció que su uso y repartición debe hacerse de manera eficaz, económica y equitativa entre los "diferentes países o grupos de países, teniendo en cuenta las necesidades especiales de los países en desarrollo y la situación geográfica de determinados países." (UIT, 1982, art. 44).

Durante esta Conferencia se hace la primera referencia al concepto de recurso órbita-espectro (ROE), que fue definido como "el recurso natural constituido por la órbita de los satélites geoestacionarios u otras órbitas de satélites, y el espectro de frecuencias radioeléctricas atribuido o adjudicado a los servicios de radiocomunicaciones por satélite por la Unión Internacional de Telecomunicaciones (UIT)" (Decisión 654 de la Comisión de la Comunidad Andina, 2006, art. 1). El ROE es diferente de acuerdo con el sistema satelital que se tenga. Es decir, cuando un satélite se encuentra en órbita geoestaciona- ria y su misión es la de ejercer funciones de teleobservación, su ROE es distinto a otro satélite que se encuentre en otro segmento de la misma órbita y que, a diferencia de este, se dedique a enviar señales de radio (Rincón, 2013, pág. 27).

\section{Reglamento de las radiocomunicaciones de la UIT de 1992}

Como se mencionó anteriormente, el Sector de Radiocomunicaciones desarrolló y adoptó el Reglamento de Radiocomunicaciones de la uाt, que consiste en un conjunto de normas que constituyen un tratado internacional vinculante, que controla la utilización del espectro radioeléctrico y las órbitas de los satélites. Este reglamento fue adoptado por más de 190 Estados Miembros y por unos 40 servicios distintos en todo el mundo, relativos a casi todos los servicios y aplicaciones terrenales y espaciales (Reglamento UIT, 1982). Este reglamento es revisado y actualizado constantemente, teniendo en cuenta que el derecho del espacio ultraterrestre es progresivo y está en permanente desarrollo gracias a las crecientes e innovadoras tecnologías. Además de las actualizaciones y desarrollos científicos y tecnológicos, este reglamento debe adaptarse a las nuevas necesidades de las telecomunicaciones. Dichas actualizaciones se llevan a cabo por medio de las Conferencias Administrativas de Radiocomunicaciones que se celebran en el marco de la uIT.

En dicho reglamento se estableció que la Oficina de Radiocomunicaciones debe procesar las asignaciones de frecuencias, al igual que la in- 
formación sobre posiciones orbitales de satélites geoestacionarios, para poder llevar a cabo las inscripciones en el Registro Internacional de Frecuencias. Para realizar la distribución de las frecuencias de una manera eficaz y efectiva se tomó la decisión de dividir al planeta en tres regiones: la Región 1 constituida por Europa, África, el Medio Oriente, Mongolia y las Repúblicas que anteriormente hacían parte de la Unión Soviética; la Región 2 conformada por países del continente americano; y finalmente la Región 3 hace referencia principalmente a Asia y Oceanía.

La distribución de las frecuencias del espectro radioeléctrico se realiza mediante un procedimiento especial dividido en tres pasos principales; (i) la atribución, (ii) la adjudicación y (iii) la asignación. La atribución hace referencia a la inscripción en el cuadro de atribución de bandas de frecuencias, con el fin de determinar su utilización en el sistema de las telecomunicaciones. El Reglamento de las Telecomunicaciones contempla dos tipos de atribución: (i) exclusiva y (ii) compartida. En la primera, la banda de frecuencias es otorgada a un único servicio de radiocomunicación, mientras que en la atribución compartida la banda de frecuencias se atribuye a dos o más servicios de radiocomunicación con el objetivo de maximizar la utilización del espectro disponible (Rincón, 2013, pág. 30).

A su vez, la adjudicación hace referencia a la inscripción de un canal determinado que va a ser utilizado por uno o varios miembros, para un servicio de radiocomunicación terrenal o espacial en uno o varios países o zonas geográficas, siguiendo unas condiciones especificadas.
Finalmente, la asignación es la autorización que da un Estado para que una estación radioeléctrica utilice una frecuencia o un canal radioeléctrico determinado. En este sentido y según explica Rincón:

Los planes de adjudicación y de asignación de frecuencias constituyen un mecanismo para preservar los derechos de los distintos estados en el acceso equitativo a recursos radioeléctricos limitados, como son el espectro de frecuencias o la órbita de los satélites geoestacionarios. (2013, pág. 30).

Ahora bien, el procedimiento para la asignación de las frecuencias se encuentra definido en el Reglamento de Radiocomunicaciones. En primer lugar, el interesado en obtener una asignación de frecuencia para un sistema satelital deberá enviar a la oficina de la UIT una descripción general de este para su publicación en la Circular Internacional de Información de Frecuencias, ${ }^{4}$ con una prelación máxima de siete años y no inferior a dos años de la fecha planeada para la entrada en servicio del sistema satelital. Si por cualquier motivo la UIT encuentra que la información presentada en la solicitud es incompleta, pedirá al interesado que la complemente o clarifique. La uIt verifica si el sistema satelital solicitado requiere de coordinación con otras administraciones, antes de notificar al solicitante sobre la aceptación de la solicitud y la asignación de la frecuencia. Si una frecuencia asignada no entra en uso dentro del término de

\footnotetext{
$4 \quad$ En caso de querer hacer alguna modificación a la propuesta enviada a la UIT para el uso de un satélite geoestacionario que modifique la posición por más de seis grados, se requerirá una nueva publicación en la Circular Internacional de Frecuencias.
} 
los siete años ${ }^{5}$ siguientes, la asignación de la frecuencia expirará.

En este Reglamento se afirma que la gestión nacional del espectro comprende las estructuras, capacidades, procedimientos y reglamentación necesaria para que cada país cumpla su objetivo de controlar la utilización del espectro radioeléctrico en su territorio y dentro de sus fronteras geográficas. Se establece que cada gobierno tiene la flexibilidad y autonomía suficientes como para regular la utilización y distribución del espectro. Bajo esta perspectiva, cada administración debe desarrollar el marco jurídico pertinente y llevar a cabo las tareas de gestión del espectro.

\section{Tratado de Nairobi de 1982}

En el Tratado de Nairobi del año de 1982 se presentaron grandes avances para el acceso a la órbita geoestacionaria y el espectro electromagnético. En el capítulo III se tratan las disposiciones especiales relativas a las radiocomunicaciones y se hace referencia a la utilización racional del espectro de frecuencias radioeléctricas y de la órbita de los satélites geoestacionarios. En el artículo 33 se afirma: “los Miembros procurarán limitar el número de frecuencias y el espectro utilizado al mínimo indispensable para asegurar el funcionamiento satisfactorio de los servicios necesarios" y se establece que los Miembros deben tener en cuenta que las frecuencias y la órbita de los satélites geoestacionarios son re-

5 Se podrán solicitar extensiones de tiempo a la uIT para el uso de la frecuencia asignada, por un periodo de tiempo que en ningún caso podrá ser superior a seis meses. cursos naturales limitados que deben utilizarse de manera eficaz y económica.

Además, se propuso hacer la asignación de las bandas de frecuencia electromagnética teniendo en cuenta las necesidades de los países en vías de desarrollo. Para esto, se le otorga a la uाт la función de fomentar la cooperación internacional en el suministro de asistencia técnica a dichos países, así como la creación, el desarrollo y el perfeccionamiento de sus instalaciones y de sus redes de telecomunicación por todos los medios de que disponga. Es decir, se reconocen las diferencias a través de la aplicación del principio de cooperación y la equidad.

De igual forma, se reafirma que la uı será la encargada de efectuar la distribución de las frecuencias del espectro radioeléctrico y llevar el registro de las asignaciones de frecuencias, a fin de evitar toda interferencia perjudicial entre las estaciones de radiocomunicación de los distintos países. Será la encargada, además, de prevenir la interferencia perjudicial entre las estaciones de radiocomunicación de los diferentes países y de mejorar la utilización del espectro de frecuencias radioeléctricas.

\section{E. Enmiendas a la Constitución de la UIT}

La Constitución y el Convenio de la uाt han sido modificados por varias enmiendas. La primera de estas tuvo lugar en la Conferencia de Plenipotenciarios de Kyoto, en 1994; la segunda en la Conferencia de Minneapolis, en el año de 1998; y la última en Marrakech ese mismo año. ${ }^{6}$

6 Según el MinTic el Estado colombiano aún no ha ratificado las modificaciones planteadas en la Conferencia de Marrakech. 
En la Conferencia de Plenipotenciarios celebrada en Minneapolis, EE. UU, en 1998, se modificó el artículo 44 de la Constitución de la uाt al establecer:

En la utilización de bandas de frecuencia para las radiocomunicaciones, los Estados miembros tendrán en cuenta que las frecuencias y la órbita de los satélites geoestacionarios son recursos naturales limitados que deben utilizarse en forma racional, eficaz y económica de conformidad con lo establecido en el Reglamento de Radiocomunicaciones, para permitir el acceso equitativo a esta órbita y a esas frecuencias a los distintos países o grupos de países, teniendo en cuenta las necesidades especiales de los países en desarrollo y la situación geográfica de determinados países.

Como se deduce del artículo 44, el Reglamento de Radiocomunicaciones, que a su vez complementa la Constitución y el Convenio de la uit, es revisado y actualizado por las decisiones de las diferentes Conferencias Mundiales de Radiocomunicaciones, de acuerdo con los avances y descubrimientos tecnológicos.

\section{NORMAS NACIONALES APLICABLES AL MANEJO DEL ESPECTRO ELECTROMAGNÉTICO Y DEL ESPECTRO RADIOELÉCTRICO}

La Constitución Política de Colombia de 1991 estableció en el artículo 75:

El espectro electromagnético es un bien público inenajenable e imprescriptible sujeto a la gestión y control del Estado. (...) Para garanti- zar el pluralismo informativo y la competencia, el Estado intervendrá por mandato de la ley para evitar las prácticas monopolísticas en el uso del espectro electromagnético.

En tal sentido, el Estado debe garantizar la igualdad de oportunidades en el acceso a dicho recurso.

El Gobierno nacional ha cumplido con lo establecido en la Constitución Internacional de Telecomunicaciones y el Reglamento de Radiocomunicaciones por medio de la Ley 52 de 1995, la Ley 514 de 1999, la Ley 873 de 2004 y la Ley 1341 de 2009. De igual forma, los decretos 4392 del 2010 y 1161 de 2010 han reglamentado la selección objetiva y las disposiciones relacionadas con el régimen de contraprestaciones en materia de telecomunicaciones.

Con la expedición de la Ley 1341 de 2009, por la cual se definieron los principios y conceptos sobre la sociedad de la información y la organización de las tecnologías de la información y las comunicaciones, se creó la ANE y se dictaron otras disposiciones, se estableció también todo lo referente a la provisión de redes y servicios, y acceso a recursos escasos en el país. En este sentido, el Ministerio de Tecnologías de la Información y las Comunicaciones (MinTic) reglamentó el procedimiento de selección objetiva para otorgar los permisos para el uso del espectro radioeléctrico a través del Decreto 4392 de 2010. En este se afirma que Colombia, a través de la Ley 671 de 2001, incorpora la asignación objetiva en lo relacionado al espectro radioeléctrico, al crear un Cuadro Nacional de Atribución 
de Bandas de Frecuencias. Este pretende regular la asignación de dichas bandas teniendo en cuenta las disposiciones del Reglamento de Radiocomunicaciones y del Convenio de la uIT.

Por otra parte, la normatividad y reglamentación del espectro radioeléctrico ha sido también materia de análisis jurisprudencial. La sentencia T-081 de 1993 define el espectro electromagnético como:

Una franja de espacio alrededor de la tierra a través del cual se desplazan ondas radioeléctricas que portan diversos mensajes sonoros o visuales. Su importancia reside en ser un bien con aptitud para transportar información e imágenes a corta y larga distancia. (CConst., 1993, E. Cifuentes).

De igual forma, la sentencia C-310 de 1996 al analizar el tema de la asignación de frecuencias establece que esta hace referencia a la autorización que da el Estado para que una estación radioeléctrica utilice una frecuencia o canal radioeléctrico determinado en condiciones específicas. El MinTic asigna las frecuencias teniendo en cuenta una solicitud previa formulada por el interesado.

Ahora bien, en el artículo 10 de la Ley 1341 de 2009 se establece que las telecomunicaciones son un servicio público bajo la titularidad del Estado. Por esta razón, debe ser el Estado, a través de la administración, el encargado de determinar el uso del espectro radioeléctrico, para garantizar a todos los ciudadanos el acceso a las telecomunicaciones. De igual forma, la sentencia C-403 de 2010 impone ciertas condicio- nes en cuanto al espectro electromagnético, en la medida en que establece que su uso "(i) debe estar coordinado con las políticas del Ministerio de Comunicaciones; (ii) no debe generar interferencias sobre otros servicios; (iii) sea compatible con las tendencias internacionales de mercado; (iv) no afecte la seguridad nacional; y (v) contribuya al desarrollo sostenible." (CConst., 403/2010, M. V. Calle).

\section{A. Agencia Nacional de Espectros}

La ANE se concibe como una unidad administrativa especial del orden nacional, adscrita al MinTic. Su objeto es brindar el soporte técnico para la gestión, planeación, vigilancia y control del espectro radioeléctrico. Fue creada mediante la Ley 1341 de 2009, y de acuerdo con el artículo 26 sus principales funciones son las de (i) asesorar al MinTic en el diseño y formulación de políticas, planes y programas relacionados con el espectro radioeléctrico y (ii) diseñar y formular políticas, planes y programas relacionados con la vigilancia y control del espectro.

Ahora bien, es el MinTic el encargado de otorgar los permisos para el uso del espectro radioeléctrico dentro del territorio nacional, en las bandas y rangos de frecuencias, según la disponibilidad y planeación del espectro. Lo anterior lo debe realizar utilizando un mecanismo de selección objetiva y siguiendo un procedimiento específico. Previo al proceso de otorgamiento del permiso de uso del espectro radioeléctrico el MinTic publicará un aviso invitando a todos los interesados a presentar manifestaciones de interés en determinada banda. 
Por otra parte, teniendo en cuenta que se requería de un sistema informático moderno que automatizara y facilitara la gestión y la comprobación técnica del espectro radioeléctrico la ANE y el MinTic crearon un Sistema de Gestión del Espectro (SGE), diseñado bajo los lineamientos de la uit con el propósito de que los proveedores de redes y servicios de telecomunicaciones puedan realizar las solicitudes para la gestión, análisis, administración y planeación del espectro radioeléctrico.

Lo anterior, según lo acordado por la República de Colombia y la uit en el Acuerdo de Cooperación Técnica 1-97, por medio del cual se adquirió una solución informática que permite automatizar la gestión del espectro radioeléctrico para todos los servicios de los recursos escasos.

La Gestión del Espectro Radioeléctrico es la combinación de los procedimientos administrativos y técnicos necesarios para garantizar la utilización eficaz del espectro de frecuencias radioeléctricas por todos los servicios de radiocomunicaciones definidos en el Reglamento de Radiocomunicaciones de la UIT, y la explotación de los sistemas radioeléctricos sin causar interferencia perjudicial. La Ley 671 de 2001, haciendo referencia a la asignación y utilización de recursos escasos, establece: “Todo procedimiento para la asignación y utilización de recursos como las frecuencias, los números y los derechos de paso se llevarán a la práctica de manera objetiva, oportuna, transparente no discriminatoria." (Anexo, numeral 6).

Por consiguiente, el SGE está dirigido a toda persona natural o jurídica debidamente constituida y domiciliada en Colombia o persona jurídica extranjera con una sucursal debidamente establecida y constituida en Colombia o proveedores de redes y servicios de telecomunicaciones establecidos con anterioridad a la Ley 1341 de 2009, que deseen solicitar permisos para el uso del espectro radioeléctrico dentro del territorio nacional en las bandas y rangos de frecuencia según la disponibilidad y planeación del espectro.

Con el SGE las personas naturales o jurídicas, proveedores de redes y servicios de telecomunicaciones podrán adelantar sus solicitudes de asignación, modificación, renovación y cancelación de frecuencias radioeléctricas, en las bandas de frecuencias que se ofertan mediante los procesos de selección objetiva que se publican en la página web del MinTic.

La ANE ha determinado la planeación estratégica del espectro, en la medida en que su objetivo principal es definir políticas, lineamientos y estrategias para el uso eficiente de este en el mediano y largo plazo, generando un impacto social positivo. Uno de los componentes requeridos para llevar a cabo una adecuada planeación y gestión del espectro es la participación internacional colombiana en el sector de radiocomunicaciones, para sincronizar los esfuerzos nacionales con iniciativas y estudios especializados que se prioricen en el marco del Ciclo de Estudios de las Conferencias Mundiales de Radiocomunicaciones de la uIT. De igual manera se espera adelantar las actividades de coordinación internacional en apoyo a la Oficina Internacional del MinTic. 


\section{CONCLUSIONES}

Teniendo en cuenta el análisis hecho anteriormente, se pude concluir que desde hace aproximadamente 145 años la uाt ha venido coordinando y reglamentando el uso y la distribución del espectro radioeléctrico a escala mundial. De igual forma, ha promovido el principio de cooperación internacional en la asignación de órbitas de satélites, ha procurado mejorar la infraestructura de telecomunicaciones en el mundo en desarrollo y ha establecido las normas mundiales que garantizan la interconexión continua de una amplia gama de sistemas de comunicaciones. Lo anterior con miras a garantizar a todos los individuos el derecho a comunicarse, para así asegurar un constante desarrollo de la sociedad.

Gracias a la indispensable labor de coordinar todos los satélites a través de la gestión del espectro y las órbitas, tenemos acceso a Internet, la televisión, la navegación por satélite, información meteorológica, cartografía en línea, etc. Es decir, la distribución y utilización eficiente del espectro radioeléctrico es indispensable en materia de telecomunicaciones. Esta función, sin duda, seguirá innovando y progresando junto con la tecnología.

De igual forma, es claro que los miembros de la Uाт han sabido adoptar sus regulaciones a la legislación nacional, con el propósito de dar un completo y eficaz cumplimiento a los principios estipulados en el Reglamento de Radiocomunicaciones, teniendo en cuenta que las órbitas de satélites y el espectro de frecuencias radioeléctricas son recursos naturales limitados que deben utilizarse en forma racional, eficaz, económica y equitativa, según lo establece el art. 44 de la Constitución de la uIT. Por lo tanto, se puede concluir que la regulación en cuanto al uso y la distribución del espectro radioeléctrico está en constante desarrollo, pues es directamente influenciada por los avances tecnológicos y científicos que, a la vez, conforman el carácter dinámico de la evolución de las telecomunicaciones.

\section{Referencias}

1. Agencia Nacional del Espectro. (2011). ¿Qué es el espectro radioeléctrico? Obtenido de ane: http://www.ane.gov.co/index. php/conozca-la-ane/que-es-el-espectro/ que-es-el-espectro.html

2. Constituyente. (1991). Constitución Política de Colombia.

3. Comisión de la Comunidad Andina. Decisión 654 de 2006. Marco regulatorio para la utilización comercial del recurso órbita-espectro de los países miembros. Noviembre 15 de 2006.

4. Corte Constitucional. Sentencia T-081 de 1993 (M. P.: Eduardo Cifuentes Muñoz; 26 de febrero de 1993).

5. Corte Constitucional. Sentencia C-310 de 1996 (M. P.: Vladimiro Naranjo Mesa; 11 de julio de 1996). 
6. Corte Constitucional. Sentencia C-403 de 2010 (M. P.: María Victoria Calle Correa; 27 de mayo de 2010).

7. Decreto 1161 de 2010 [Ministerio de Tecnologías de la Información y las Comunicaciones]. Por el cual se dictan algunas disposiciones relacionadas con el régimen de contraprestaciones en materia de telecomunicaciones y se derogan los decretos 1972 de 2003 y 2805 de 2008. Abril 13 de 2010.

8. Decreto 4392 de 2010 [Ministerio de Tecnologías de la Información y las Comunicaciones]. Por el cual se reglamenta la selección objetiva y la asignación directa por continuidad del servicio de que tratan los artículos 11 y 72 de la Ley 1341 de 2009. Noviembre 23 de 2010.

9. Ley 514 de 1999. Por medio de la cual se aprueban las "Actas Finales de la Conferencia Mundial de Radiocomunicaciones" (CMR95), reunida en Ginebra del veintitrés (23) de octubre al diecisiete (17) de noviembre de mil novecientos noventa y cinco (1995). Agosto 4 de 1999. DO n. ${ }^{\circ} 43656$.

10. Ley 671 de 2001. Por medio de la cual se aprueba el "Cuarto Protocolo anexo al Acuerdo General sobre el Comercio de Servicios con la Lista de Compromisos Específicos de Colombia Anexa", hecho en Ginebra el 15 de abril de 1997.

11. Ley 873 de 2004. Por medio de la cual se aprueban el Instrumento de Enmienda a la
Constitución de la Unión Internacional de Telecomunicaciones (Ginebra, 1992), con las enmiendas adoptadas por la Conferencia de Plenipotenciarios (Kyoto, 1994) (Enmiendas adoptadas por la Conferencia de Plenipotenciarios (Minneápolis, 1998), firmado en Minneápolis, el seis (6) de noviembre de mil novecientos noventa y ocho (1998), y el Instrumento de Enmienda al Convenio de la Unión Internacional de Telecomunicaciones (Ginebra, 1992), con las enmiendas adoptadas por la Conferencia de Plenipotenciarios (Kyoto, 1994), (Enmiendas adoptadas por la Conferencia de Plenipotenciarios (Minneápolis, 1998), firmado en Minneápolis, el seis (6) de noviembre de mil novecientos noventa y ocho (1998). Enero 5 de 2004. DO n. ${ }^{\circ}$ 45421.

12. Ley 1341 de 2009. Por la cual se definen principios y conceptos sobre la sociedad de la información y la organización de las Tecnologías de la Información y las Comunicaciones -TIC-, se crea la Agencia Nacional del Espectro y se dictan otras disposiciones. Julio 30 de 2009. DO n. ${ }^{\circ} 47426$.

13. Rincón Cortés, C. (2013). Aspectos jurídicos relativos a la utilización del espectro electromagnético y radioeléctrico y su importancia en las telecomunicaciones por satélite. Revista de Derecho Público, (30).

14. Unión Internacional de Telecomunicaciones [UIT]. (1965). Convenio Internacional de Telecomunicaciones (Montreux). Ginebra: Secre- 
taría General de la Unión Internacional de Telecomunicaciones.

15. Unión Internacional de Telecomunicaciones. (1967). Tratado sobre los principios que deben regir las actividades de los Estados en la exploración y utilización del espacio ultraterrestre, incluso la luna y otros cuerpos celestes. Washington: Naciones Unidas.

16. Unión Internacional de Telecomunicaciones. (1973). Conferencia de Plenipotenciarios de la Unión Internacional de Telecomunicaciones (UIT) de Málaga-Torremolinos.

17. Unión Internacional de Telecomunicaciones. (1982). Tratado de Nairobi. Ginebra: Secretaría General de la uıт.
18. Unión Internacional de Telecomunicaciones. (1985). Reglamento de Radiocomunicaciones. Ginebra: uIt.

19. Unión Internacional de Telecomunicaciones. (1998). Conferencia de Plenipotenciarios. Minneapolis, EE. UU.: UIT

20. Unión Internacional de Telecomunicaciones. (2010). Comisiones de Estudio del UIT-R. Ginebra, Suiza: Oficina de Radiocomunicaciones de la uit. Obtenido de itu.int: http://www. itu.int/dms_pub/itu-r/oth/OA/OE/ROAOE0000010001PDFS.pdf 